\title{
Forecasting Fire Growth using an Inverse CFD Modelling Approach in a Real-Scale Fire Test
}

\author{
WOLFRAM JAHN, GUILLERMO REIN, and JOSÉ L. TORERO \\ BRE Centre for Fire Safety Engineering \\ The University of Edinburgh \\ Edinburgh, EH9 3JL, United Kingdom
}

\begin{abstract}
The rate of fire growth in a real-scale fire test is estimated using an inverse modelling approach of computational fluid dynamics (FDS v5). Measurements from the fire test are assimilated into the fire model and based on this the parameters of a decoupled fire growth model are estimated. A forecast of the fire development is then made with the estimated parameters. It is shown that a simplified fire growth model can give a robust representation of the underlying physics and that the necessary parameters can be estimated at an acceptable computational cost. The forecasts are shown to accurately predict the fire development. The results are based on a simplified single parameter fire growth model of a well characterized scenario, but the methodology allows for an extension to a more complicated model that would require less previous characterization of the fire scenario.
\end{abstract}

KEYWORDS: fire dynamics, forecast, CFD.

\section{INTRODUCTION}

Forecasting fire dynamics is conjectured to lead to a paradigm shift in the response to fire emergencies in buildings, providing the fire service with essential information about the development of the fire with some lead time (i.e. seconds or minutes ahead of the event) [1]. An attempt to apply a forecasting methodology to fire dynamics of a real fire is presented in this paper using the inverse model methodology developed in Ref. [2], and using the real-scale experiment of Dalmarnock Test One. The spread rate of a fire on a burning sofa in a full scale compartment is estimated using an inverse modelling approach, and a forecast of the fire development is made.

The term 'forecast' is used in the context of this paper as a theoretical process of predicting fire development based on comparison to observations from the evolving fire scenario, i.e. based on (automatic) tuning of the model with information received from observations. In reality positive lead times can not yet be provided if numerical fire models are used for the forecast, as the assimilation process alone still takes longer than a typical fire scenario (15-20 min assimilation compared to 5 min fire), despite important simplification and resulting speed-up (see Ref. [2]).

The experimental set up, procedure and results of the Dalmarnock Tests are presented elsewhere [3,4], and will not be discussed here.

In Ref. [5] the early HRR development of Dalmarnock Test One, which had not been measured during the test, was estimated by comparing field model output to observations from the test. Based on laboratory experiments, two HRR curves were established that provided an upper and a lower bound for a possible HRR. Although the modelling work was done a posteriori, it suggested that CFD type modelling could be used to recover to a certain extent the underlying physics of the fire growth in a real compartment fire. However, the lead time of that approach was in the order of months (that is the time it took to establish the HRR bounds), which is far from a positive lead time.

\section{Simple Extrapolation}

The technology of forecasting fire dynamics based on numerical simulation of the underlying physics is currently limited because available fire simulation tools cannot predict fire growth in a fast, precise and robust manner. 
Nevertheless, a very simple and fast way of forecasting fire development is to analyze the time history of the average temperature in the compartment (which can be estimated from live observations from the fire scenario). An adequate curve can be fitted to the available data and, assuming that the temperatures grow following the same curve, it is possible to forecast the average temperature with some acceptable uncertainty. This method involves no physics or fundamental knowledge.

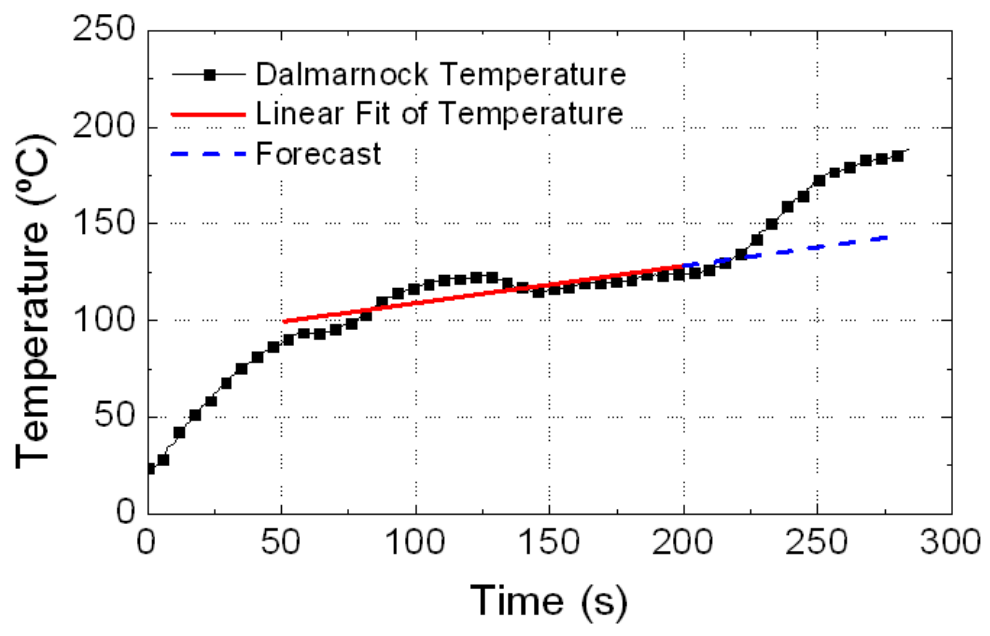

Fig. 1.Forecast of real fire test data based on simple curve fitting.

Figure 1 shows the average compartment temperature during the first $300 \mathrm{~s}$ of Dalmarnock Test One. After an initial rapid increase the temperatures follow a relatively slow linear growth until about $220 \mathrm{~s}$. If a forecast was made $200 \mathrm{~s}$ after ignition, the temperatures would be well approximated by a linear fit as indicated in Fig. 1. Due to the decoupling from the underlying physics, the forecast can not anticipate changes in the growth pattern and is limited to short times (in this case until $220 \mathrm{~s}$ ). The usefulness of this approach for assisting the decision making process of commanding fire service officers is equally limited, especially considering that the forecast is only valid for those sensor locations whose readings are included in the observations (if thermocouples are only installed close to the ceiling, the forecasted development is only for temperatures close to the ceiling, not for general temperatures). However, the forecast is almost instantly available without the use of extensive computational resources, and any more sophisticated forecasting system will have to outperform this approach (at least in some way) in order to be considered for implementation.

The methodology presented in Refs. [2,6] if applied to real data could provide a useful, physics based forecast within a reasonable time frame.

A simplified and decoupled fire growth model, as presented in Refs. [2,6], is thus implemented, estimating the spread rate of the fire on the sofa. Other parameters necessary for the simulation of a complex fire scenario such as Dalmarnock Test One were taken from previous analysis [2,7] and are assumed to be known. The sensitivity of these parameters is discussed in detail in Ref. [7].

\section{PRIOR ANALYSIS}

The ignition and early burning of the sofa in the Dalmarnock Fire Tests was reproduced in several laboratory experiments in order to provide an input HRR for numerical modelling of the Tests, see Refs. $[2,5]$. The first of these experiments was conducted before the Dalmarnock Test series was carried out, and the ignition protocol differed somewhat from the protocol finally adapted in Dalmarnock. In the laboratory experiments the ignition source (a basket filled with crumpled waste paper and some accelerant) was standing next to the sofa without direct contact to the sofa, whereas in Dalmarnock both items (bin and sofa) were connected by means of a blanket placed over the armrest and hanging into the waste paper 
basket. Accelerant had been spilled in the bin and on the blanket in order to assure reasonably rapid ignition of the seating area of the sofa.

A second series of laboratory experiments was conducted after the Dalmarnock Tests. In these experiments the ignition protocol was reproduced as closely as possible based on layout information, photographs and video recordings from Dalmarnock.

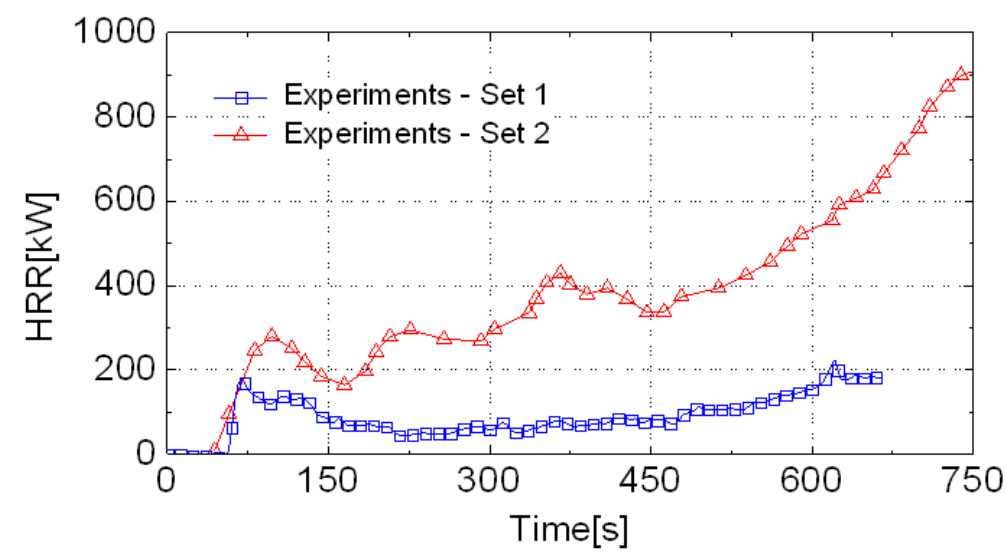

Fig. 2. HRRs for sofa as measured in laboratory experiments.

Figure 2 shows the HRRs as estimated using oxygen depletion calorimetry in the laboratory experiments. The blue data correspond to the first experiment (Set 1, carried out before the Dalmarnock Tests), while the red correspond to the second series of experiments (Set 2, carried out after the Tests). The blanket, included in Set 2, is conjectured to have acted as a bridge between the burning bin and the seating area of the sofa, bypassing the barrier of the armrest. The fire could thus spread to the seating area of the sofa more easily.

The HRR curves presented in Fig. 2 show two distinct patterns in time (this is discussed with more detail in Ref. [2]). The initial peak, that can be attributed to the burning of the waste paper bin, is followed by a slower growth which is conjectured to correspond to the sofa itself. The growth on the sofa can be approximated to good agreement by a quadratic curve.

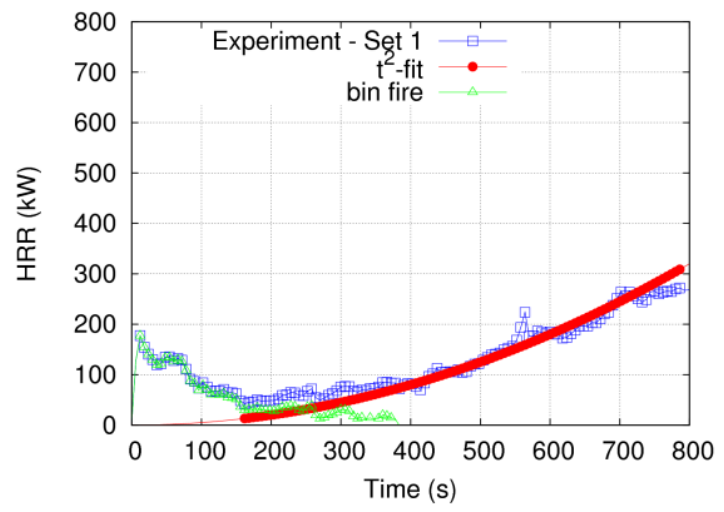

(a)

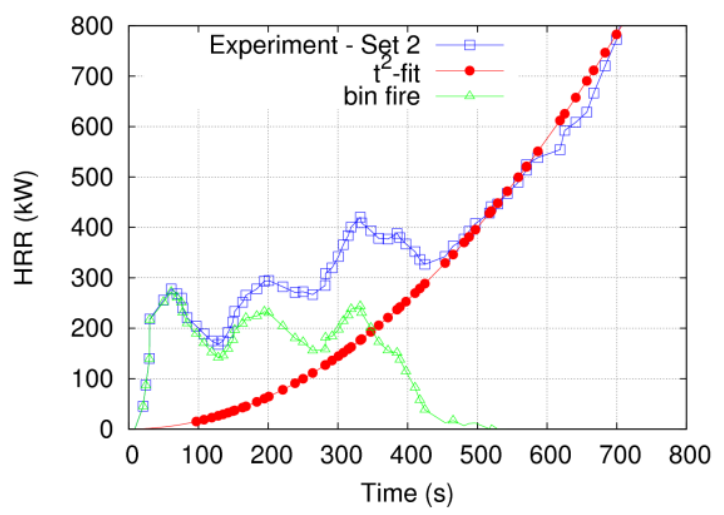

(b)

Fig. 3. Quadratic fit of the HRR curves measured in the laboratory experiments with estimated bin fire: (a) experiment prior to Dalmarnock (Set 1 according to Ref. [2]); (b) second series of experiments, conducted after Dalmarnock (Set 2 according to Ref. [2]).

Figure 3 shows the HRRs with their respective $t^{2}$-fit and the HRR of the waste paper bin together with the blanket (which is obtained by subtracting the $\mathrm{t}^{2}$-fit from the original data). The contribution of the bin fire is 
negligible after about $400 \mathrm{~s}$ in both cases. Note that there is considerable uncertainty in the characterisation of the bin fire, both quantitatively and qualitatively.

The $\mathrm{t}^{2}$-curve that can be fitted to both laboratory experiments confirms the validity of a fire growth consistent with the growth model proposed in [2], where the HRR is assumed to be proportional to the fire area,

$$
\dot{Q}=\Delta h_{c} \dot{m}=\Delta h_{c} \dot{\omega} A(r, t) .
$$

Here $\Delta h_{c}$ is the effective heat of combustion (in $\mathrm{kJ} / \mathrm{kg}$ ), $r(\mathrm{~m} / \mathrm{s}$ ) is the spread rate of the fire (assumed constant), and $\dot{\omega}\left(\mathrm{kg} / \mathrm{s} \cdot \mathrm{m}^{2}\right)$ is the fuel burning rate per unit area. As long as the fire does not reach the boundaries of the fuel surface the fire area is circular $\left(A(r, t)=\pi\left(r\left(t-t_{0}\right)\right)^{2}\right)$, and the resulting HRR follows a quadratic growth.

The fire spread model presented in Eq. 1 is thus used in this approach to characterize the fire growth, and the growth rate is estimated.

\section{MODEL DETAIL}

The fire specific CFD code Fire Dynamics Simulator version 5.1.6 (FDS v5) [8] is used for the fire simulations in the forward model. FDS solves a form of the Navier-Stokes equations adequate for lowspeed thermally driven flows.

\section{Computational Domain}

Detailed information about the experimental set-up of Test One can be found elsewhere [3,4]. Test One was held in a two-bedroom single family flat, with the living room set up as the main experimental compartment.

In order to make the modelling more efficient in terms of computational time, the computational domain concentrated on the main compartment room of Dalmarnock Test One and its vent openings (Fig. 4). This compartment was $3.50 \mathrm{~m}$ by $4.75 \mathrm{~m}$ wide and $2.45 \mathrm{~m}$ high with a $2.35 \mathrm{~m}$ by $1.18 \mathrm{~m}$ two-pane window. A detailed account of the model set up is given in [2].

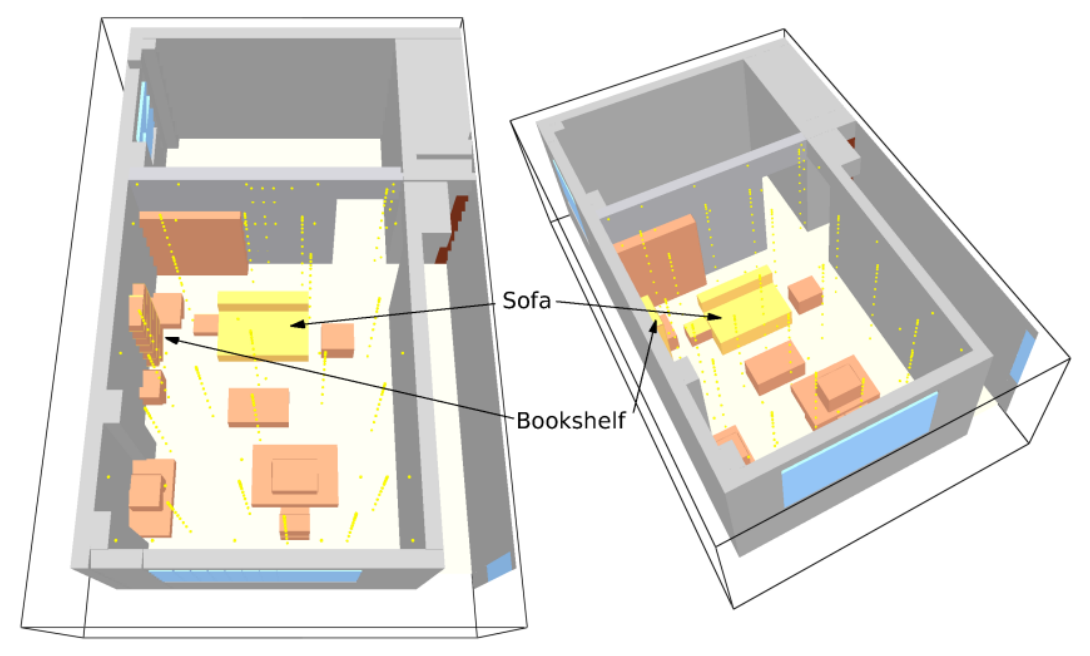

Fig. 4. Computational domain and fuel items used in the FDS simulations. 
For eventual fluid dynamic effects on the ventilation flows, the kitchen and an artificial hallway adjacent to the main compartment are included as shown in Fig. 4. The other rooms of the apartment were not considered, since they did not contribute to the fuel load nor significantly affected the ventilation flows.

The fuel load in the main compartment included the sofa and two bookshelves in the corner behind the sofa as shown in Fig. 4. Although the coffee table, chairs and desks also contributed to the fuel load, this occurred only after flashover, and will not be considered here.

Figure 5 shows a plan view of the main experimental compartment. The blue circles represent thermocouple trees. Each of the trees had 12 thermocouples, distributed along its height, as described in detail in [3].
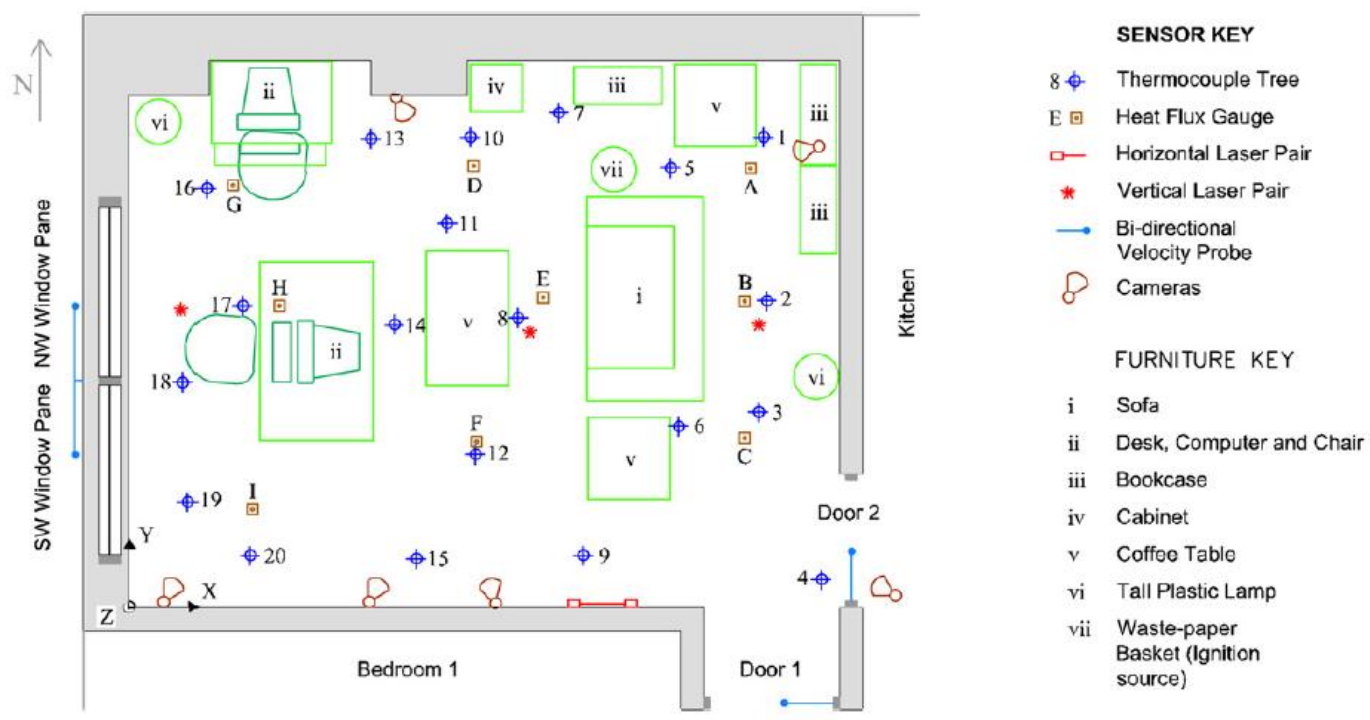

Fig. 5. Plane view of the experimental compartment.

\section{Grid Size}

A grid sensitivity study for FDS applied to the Dalmarnock Test One was conducted by Jahn et al. [2,5], where it was shown that the results from a simulation with $20 \mathrm{~cm}$ grid are still in good agreement with the experiments, although the plume is not well resolved. In the context of this work computational speed is the primary goal, as details that are lost by the insufficient numerical resolution can be recovered from observations from the fire, and a $20 \mathrm{~cm}$ grid is thus chosen for the forward model simulations.

\section{Modelling of the Fire}

According to the analysis presented above, the fire is modelled in a twofold way. The area of the bin fire is assumed as constant, and its intensity is modelled as a constant HRR per unit area that decreases linearly to zero between $150 \mathrm{~s}$ and $250 \mathrm{~s}$. This is a crude approximation of the contribution of the bin to the total HRR based on the data presented in Fig. 3a and Fig. 3b. The fire growth on the sofa is modelled by subsequent ignition of surface cells according to a prescribed spread rate, starting from a point on the edge of the sofa closest to the burning bin (at the same time the bin ignites). By doing this the fire spread is decoupled from the heat feedback from the flame, and the spread rate becomes the invariant parameter to estimate.

Exact modelling of the fire spread over the sofa is not possible with this approach, due to the complicated geometry of the sofa that can not be captured by the coarse grid. It must be kept in mind however that the exact dimensions of the fuel source are not generally known to the forecasting system, and it is more realistic to assume some kind of equivalent fuel (the determination of a general equivalent fuel load that represents the greatest possible number of fuel sources is as yet a pending issue). In the present case it was 
assumed that the sofa is a plane surface of $1.44 \mathrm{~m}^{2}$, which corresponds to the sum of the areas of the sofa's different surfaces (within the restriction that it has to be consistent with the grid). The sofa (i.e. the plane surface that represents it) in the forward model is located in approximately the same place as in the real scenario.

\section{Data Assimilation}

Data is assimilated following the methodology proposed in Ref. [2]. A summary of the methodology is presented in the following paragraphs.

The problem to be solved is the correct estimation of a set of parameters, which characterize the decoupled fire growth model. These parameters can include the spread rate(s) of the fire(s), burning rate(s), radiative fraction(s) etc, and they are assumed not to change their value for a significant range of time (until a change in the ventilation conditions for example). The problem can thus be represented on the basis of these invariants summarized in the vector $\boldsymbol{\theta}$,

$$
\theta=\left[r_{1}, r_{2}, \ldots, \omega_{1}, \omega_{2}, \ldots, \chi_{1}, \chi_{2}, \ldots\right]
$$

Once a fire has been detected and observations are collected during the assimilation window, the relevant invariants in $\boldsymbol{\theta}$ can be estimated and a forecast of the fire development can be made without solving the complex interactions between gas and solid phase. The assimilation window is the period of time where observations are received and considered for the optimization step. As time goes by, new observations come in, providing more information on the history of the fire development.

Data are assimilated into the model by minimizing a cost function that describes the distance between the model output and the measurements. The governing parameters are then adjusted until convergence is obtained.

$J(\theta)=\sum_{i}^{N}\left[y_{i}-\hat{y}_{i}(\theta)\right]^{T} W_{i}\left[y_{i}-\hat{y}_{i}(\theta)\right]$

where $y_{i}$ is the set of physical variables that is measured, and $\hat{y}_{i}(\theta)$ is the output of the forward integration of the numerical model that computes the state of the system at time $t_{i}$ from the initial state $y_{0}$, and $W_{i}$ is a weight matrix. The parameters to be estimated are denoted by the vector $\boldsymbol{\theta}$. Data is assimilated during the assimilation window that is discretized according to the output of the numerical model in $N$ time steps.

The inverse problem that has to be solved in order to estimate the parameters $\boldsymbol{\theta}$ can be formulated as the following minimization problem:

$$
\min _{\theta} J(\theta)
$$

s.t. $\quad \hat{y}_{i}(\theta)=M_{i}\left(y_{0}, \theta\right)$,

where $M_{i}\left(y_{0}, \theta\right)$ denotes the forward integration model (the fire specific CFD code FDS in this case).

Due to the high computational cost of function evaluations, a gradient based optimization method was chosen. The gradient of the cost function, which includes the gradient of the forward integration model, is obtained by means of a finite difference approach.

The high density of instrumentation in the Dalmarnock Tests and the relatively fine grid of gas phase temperature measurements provide a fairly high number of observations that can be assimilated into the forecasting system. And although it might be argued that a sensor distribution as uniform as seen in the Dalmarnock Tests is not realistic, those sensors that provide the most useful information during the early stages of the fire are the ones close to the ceiling, where the highest temperatures are recorded. Therefore using only sensors close to the ceiling might be sufficient (which is feasible from an operational point of 
view). In this attempt of using CFD to forecast fire dynamics of a real-scale fire test, gas phase temperature observations at locations higher than $1 \mathrm{~m}$ were assimilated.

\section{Forecast Results}

The parameter to be estimated was the spread rate of the fire on the sofa. The bin fire was defined based on the results presented in Fig. 2, as mentioned above. All other input parameters that needed to be defined in order to produce a forecast (radiative fraction, soot yield etc) of the fire were set to values within the ranges given in Ref. [7] or to default FDS v5 values if applicable. Two hundred seconds of data were used for assimilation.

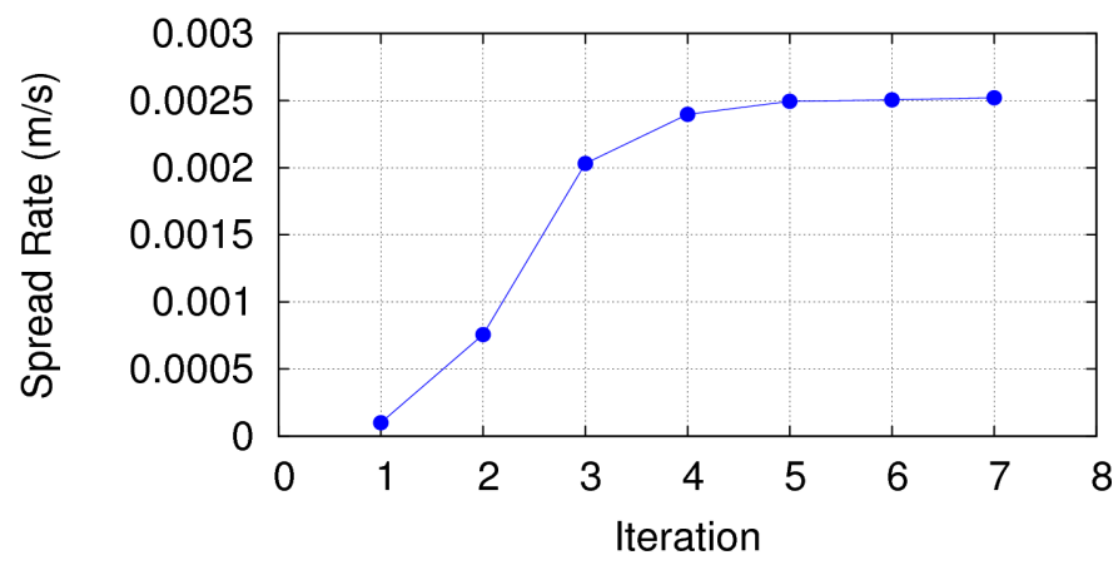

Fig. 6. Convergence of the spread rate as function of iterations.

Figure 6 shows the estimated spread rate as a function of the iterations. The process is started with only a bin fire (spread rate on the sofa is set to zero). After four iterations it has converged to $2.5 \mathrm{~mm} / \mathrm{s}$.

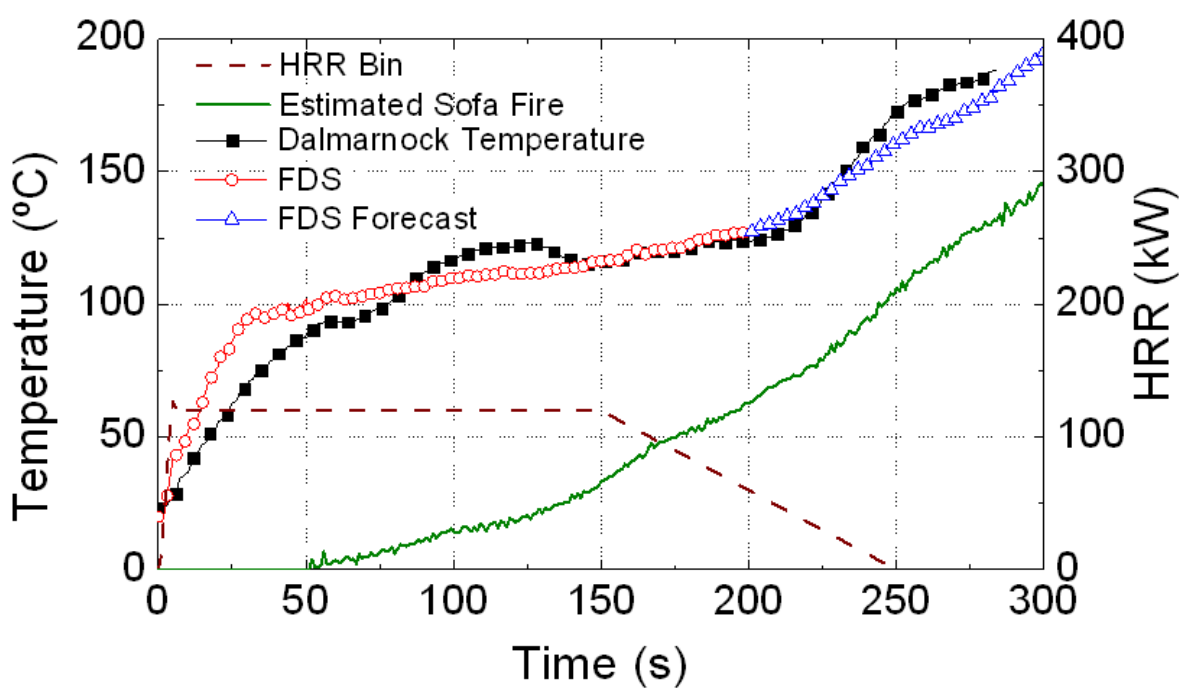

Fig. 7. Forecast of fire development in Dalmarnock Test One.

The result of the analysis is shown in Fig. 7, where a forecast is made with a spread rate of $2.5 \mathrm{~mm} / \mathrm{s}$ over the sofa. The temperature presented is an average of all the temperature observations considered for assimilation. The solid squares represent the observations from Dalmarnock Test One, while the circles are the model output within the assimilation window (less than $200 \mathrm{~s}$ ). The triangles represent the forecast of 
the average temperature made based on the information of the assimilated data. The total HRR of the forecast is a combination of the bin fire (dashed line) and the estimated sofa fire (continuous line). The upwards trend of the observed temperature in Dalmarnock after $220 \mathrm{~s}$ is forecasted correctly.

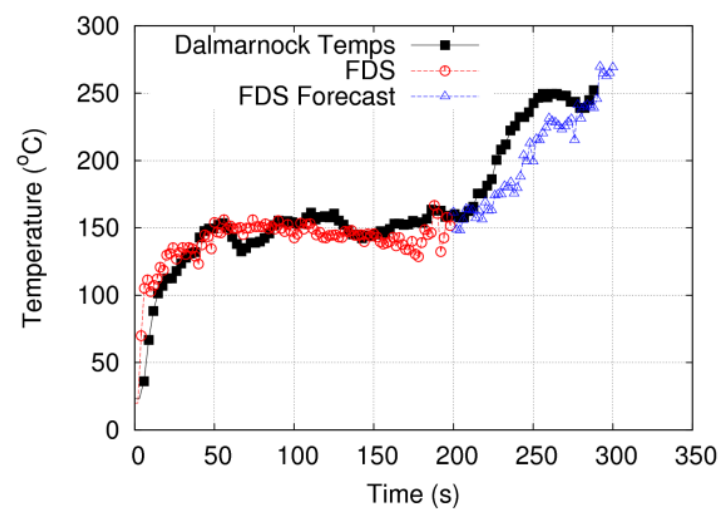

(a)

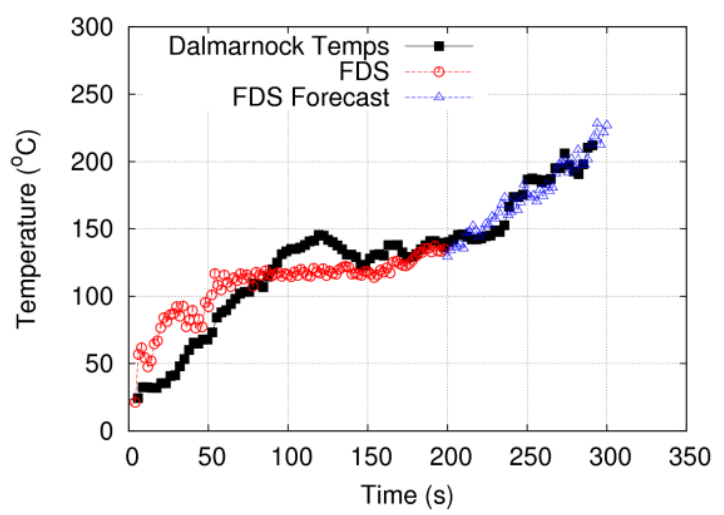

(b)

Fig. 8. Forecast of the temperature at Rack 1 (see Fig. 5 for sensor location): (a) at $230 \mathrm{~cm}$ above floor level (15 cm from ceiling); (b) at $160 \mathrm{~cm}$.

The temperature forecasts at six localized points of comparison are presented in the following sections. Figure 8 shows the forecast of temperature development in the north east corner of the compartment (thermocouple rack 1, see Fig. 5). Although the increase in temperature after $200 \mathrm{~s}$ is forecasted with a slightly less steep slope at $230 \mathrm{~cm}$ above floor level, the differences between true development and forecast remain within $10 \%$. At the thermocouple at $160 \mathrm{~cm}$ above the floor the forecasted temperatures show the same growth rate as the true temperatures.

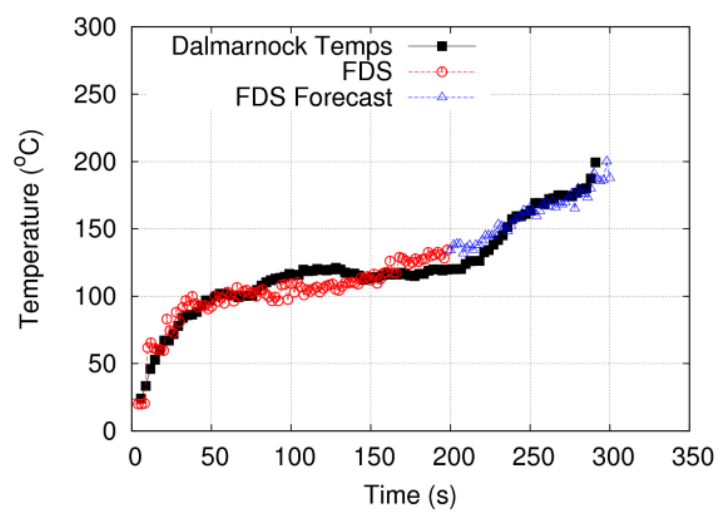

(a)

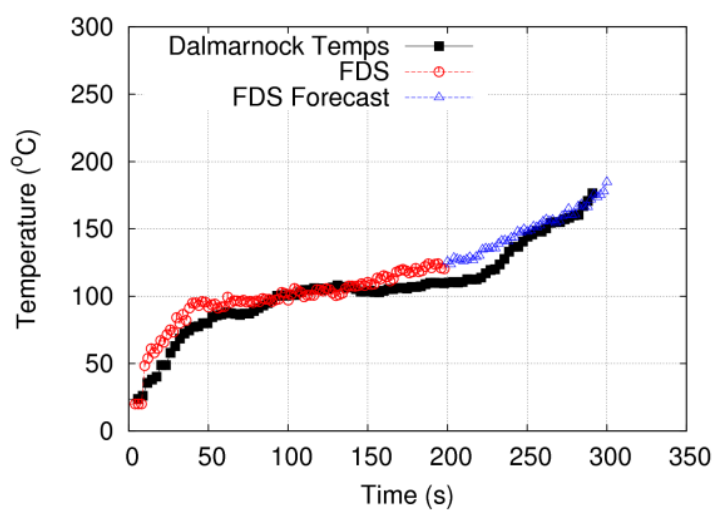

(b)

Fig. 9. Forecast of the temperature at Rack 19 (see Fig. 5 for sensor location): (a) at $230 \mathrm{~cm}$ above floor level (15 cm from ceiling); (b) at $160 \mathrm{~cm}$.

Figure 9 shows the temperature development at rack 19 in the south west corner of the compartment, close to the window (see Fig. 5 for exact location). At $230 \mathrm{~cm}$ above the floor the forecasted temperature rise coincides with the true development, providing good agreement until $300 \mathrm{~s}$. At $160 \mathrm{~cm}$ above floor level the temperature is slightly overpredicted during the assimilation period (until $200 \mathrm{~s}$ ), but the forecast is within $10 \%$ of the true development. 


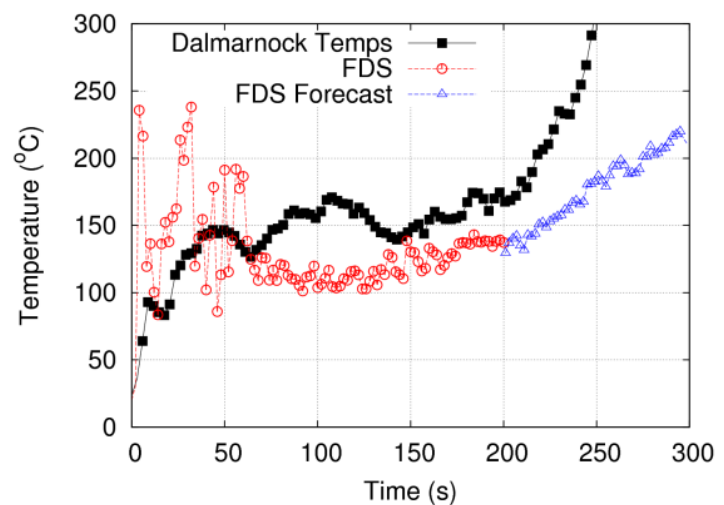

(a)

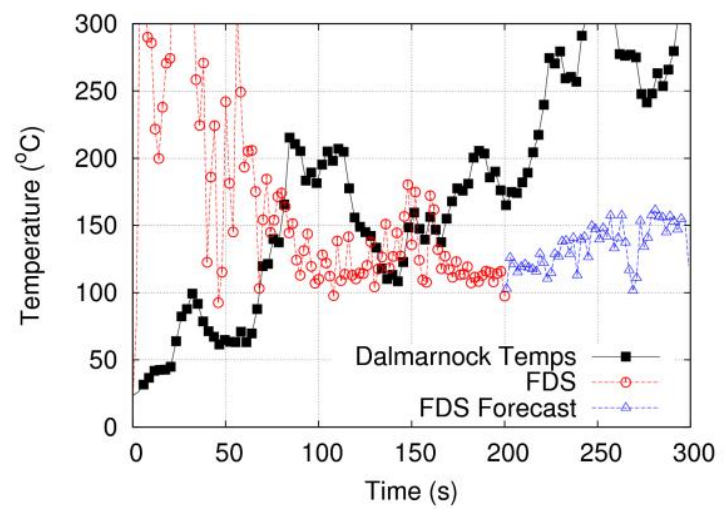

(b)

Fig. 10. Forecast of the temperature at Rack 7 (see Fig. 5 for sensor location): (a) at $210 \mathrm{~cm}$ above floor level (35 cm from ceiling); (b) at $140 \mathrm{~cm}$.

Close to the flaming region the forecast is expected to be of less quality, due to the low resolution of the numerical grid. Figure 10 shows the temperature development at rack 7 located next to the burning waste paper basket. Especially during the early stages of the fire, when the contribution of the basket is considerable and combustion takes place in the cells where the thermocouples are located, the temperatures produced by FDS show great fluctuations and do not agree well with the data from the true fire. The forecasted temperatures present a lower rate of increase compared to the true temperature development.

\section{CONCLUSIONS}

It was possible to find a good fit between the observation and the simulations, and estimate a spread rate that lies within the possible range of values. A forecast of future fire development could be made with a lead time of around $50 \mathrm{~s}$.

A realistic fire spread rate could be estimated based on comparison of model output to measurements of the fire scenario, although it could not be compared to the true value, as no estimation of the spread rate on the sofa was made in the Dalmarnock Tests.

A forecast of a real fire event could be made with a potentially longer lead time than a forecast based on the gradient of the temperature measurements (if, of course, it is assumed that the assimilation time is negligible). While the temperature gradient method predicts a constant growth of $0.19{ }^{\circ} \mathrm{C} / \mathrm{s}$ after $200 \mathrm{~s}$, the method presented is able to predict the change in the gradient after $220 \mathrm{~s}$.

Another advantage of this method over the curve fit forecast presented in Fig. 1 is that the CFD based forecast is also able to predict localized temperatures, not only average. Here the real strength of the proposed forecast methodology was shown. The forecasted temperature at localized points in the compartment was shown to be in very good agreement with the true development of the temperatures, as long as measurements were compared outside the flaming region. Within the flaming region the forecast is, as expected, of much less quality. Although only a few points of comparison are shown here, the good agreement of the forecast with the true temperature development was generally observed throughout the compartment. Thus, using this CFD based forecast methodology the temperature at locations away from measurements can be extrapolated, and the spatial distribution of temperatures in the room can be forecasted.

Many issues have still to be addressed. Dalmarnock Test One is a very challenging set of data for the tested methodology. The predominance of the bin fire in the early stages of the fire makes it difficult to correctly estimate the spread rate of the fire on the sofa. Indeed, assuming a different burning pattern for the bin fire (variable instead of constant) would change the estimation of the spread rate of the fire on the sofa, and the robustness of the method has to be investigated in this context. 
Another pending matter is the fact that the assimilation time with this forecasting methodology is still not negligible. Although the CFD model could be run with a very coarse grid, it took between 15 to 20 min to estimate the parameters correctly and to produce a forecast. Compared, however, to run times that would be required by traditional CFD based fire spread models with no information feedback from the fire scenario, these results are very close to positive lead times, and it is conjectured by the author that positive lead times will be possible in the near future with this methodology.

\section{REFERENCES}

[1] Cowlard, A., Jahn, W., Abecassis-Empis, C., Rein, G. and Torero, J.L., (2010) Sensor Assisted Fire Fighting, Fire Technology 46(3): 719-741. http://dx.doi.org/10.1007/s10694-008-0069-1

[2] Jahn, W., "Inverse Modelling to Forecast Enclosure Fire Dynamics", The University of Edinburgh, PhD thesis, Edinburgh, 2010. http://www.era.lib.ed.ac.uk/handle/1842/3418

[3] Abecassis-Empis, C., Reszka, P., Steinhaus, T., Cowlard, A., Biteau, H., Welch, S. and Rein, G., (2008) Characterisation of Dalmarnock Fire Test One, Experimental Thermal and Fluid Science 32(7):1334-1343. http://dx.doi.org/10.1016/j.expthermflusci.2007.11.006

[4] The Dalmarnock Fire Tests: Experiments and Modelling, $1^{\text {st }}$ edition, ISBN 978-0-9557497-0-4, 2007, Accessible at www.era.lib.ed.ac.uk/handle/1842/2037

[5] Jahn, W., Rein, G. and Torero, J., (2011) A Posteriori Modelling of the Growth Phase of Dalmarnock Fire Test One, Building and Environment, 46:1065-1073. http://dx.doi.org/10.1016/j.buildenv.2010.11.001

[6] Jahn, W., Rein, G. and Torero, J.L. (2011), Forecasting Fire Growth using an Inverse Zone Modelling Approach, Fire Safety Journal, 46: 81-88. http://dx.doi.org/10.1016/j.firesaf.2010.10.001

[7] Jahn, W., Rein, G. and Torero, J.L., (2008) The Effect of Model Parameters on the Simulation of Fire Dynamics, Fire Safety Science, 9:1341-1352. http://dx.doi.org/10.3801/IAFSS.FSS.9-1341

[8] McGrattan, K., Rehm, R. and Baum, H., (1998) Large Eddy Simulations of Smoke Movement, Fire Safety Journal 30(2): 161-178. http://dx.doi.org/10.1016/S0379-7112(97)00041-6 